Jóvenes rurales y transiciones postsecundarias:

Expectativas y estrategias para el acceso

a la educación superior

Young rural people and postsecondary transitions: expectations and strategies for accessing

higher education

\author{
Melissa Villegas Quispe \\ Instituto de Estudios Peruanos, \\ Pontificia Universidad Católica del Perú. \\ melissa.villegas@pucp.pe
}

Recibido: 26-4-2016

Aprobado: 18-7-2016 


\title{
Resumen
}

El presente artículo expone los hallazgos de un estudio reciente sobre un grupo de jóvenes del sur andino del Perú, quienes acababan de culminar la secundaria. El estudio aborda sus expectativas y estrategias para desarrollar sus proyectos de vida poniendo énfasis en sus posibilidades y limitaciones para alcanzar los estudios superiores, meta deseada por la mayoría. Desde un enfoque de curso de vida, se analizaron las transiciones postsecundarias y se encontró que las expectativas y estrategias educativas están mediadas por una compleja combinación de factores económicos, culturales, y educativos que posibilitan y limitan las transiciones de los jóvenes. Mediante la reconstrucción de historias de vida, este estudio busca comprender el proceso que los jóvenes rurales de un contexto particular experimentan una vez que culminan su escolaridad.

Palabras clave: jóvenes rurales, transiciones postsecundarias, expectativas, estrategias, Perú

\begin{abstract}
This paper presents the findings of a recent study about a group of young people from the central Peruvian Andes who finished secondary school the previous year. The study focuses on young people's expectations and strategies to develop their life projects. It emphasizes their possibilities and limitations to reach higher education, the goal of most of them. From a life course approach, it analyses the postsecondary transitions and shows that expectations and educational strategies are mediated by a complex combination of economic, cultural and educational factors that both enable and constrain youth transitions. By reconstructing life histories, this study seeks to understand the process of rural young people, in a particular Andean and rural context, once they finished their secondary school.

Keywords: young rural people, postsecondary transitions, expectations, strategies, Peru
\end{abstract}




\section{Jóvenes rurales y transiciones postsecundarias: Expectativas y estrategias para el acceso a la educación superior ${ }^{1}$}

\section{Introducción}

La finalización de la educación secundaria y el tránsito a los estudios superiores son dos fenómenos crecientes, que cobran mayor notoriedad en zonas rurales del país donde, unas décadas atrás, eran escasas las oportunidades educativas para las generaciones jóvenes. En el contexto actual, se ha generado una mayor oferta y demanda de servicios educativos públicos y, sobre todo, privados. Ello representa una oportunidad más cercana y local para las recientes generaciones de jóvenes que buscan ser profesionales y quienes, en décadas anteriores, hubiesen tenido que abandonar sus localidades para alcanzar dicho objetivo.

Sobre este panorama, muchos jóvenes experimentan la educación secundaria como un período de preparación para el acceso a los estudios superiores principalmente universitarios. Dado que existe una intención de prolongar las trayectorias educativas al nivel superior, durante su paso por la secundaria, es central la elaboración de un proyecto de vida cuyo principal objetivo sea llegar a ser profesional. Si bien el tránsito a los estudios superiores forma parte de las expectativas de los jóvenes y sus familias, también, representa una inversión adicional a la que no todos pueden acceder. Por ello, el final de la secundaria se convierte en un momento clave para los jóvenes, porque no solo potencia sus expectativas y moviliza estrategias para asegurar el acceso a la educación superior, sino también se constituye como una etapa en la que se lidia con la incertidumbre que puede interrumpir y transformar las trayectorias educativas de estos actores.

En este artículo, se mostrarán los hallazgos de una investigación sobre las expectativas y estrategias de un grupo de jóvenes, varones y mujeres, durante la transición postsecundaria. Este estudio se realizó en el año 2012 en la provincia de Andahuaylas en la región Apurímac. En este, participaron 20 jóvenes que culminaron la secundaria en el año 2011 y quienes provienen de familias en las que nunca antes hubo profesionales.

La investigación se enmarca en el campo de estudio sobre juventud, dentro del enfoque de curso de vida, que se ha venido desarrollando a partir de la década de 1970 en Estados Unidos y Europa (Elder, 1994; Ulrich, 2004; Abbott, 1997; Heinz \& Krüger, 2001). En Latinoamérica, también existen estudios similares relacionados con el papel de la educación y el trabajo en los jóvenes (Dávila, 2005; Sepúlveda, 2013; Otero, 2010). El curso de vida es un enfoque teórico-metodológico que integra la construcción individual con las condiciones estructurales y que, por tanto, hace posible comprender los distintos

1. Este artículo es parte de mi tesis de licenciatura en Antropología por la Pontificia Universidad Católica del Perú, que fue financiada por el Vicerrectorado de Investigación de la misma universidad. 
cambios que adoptan los sujetos en relación con su edad y, a lo largo de su vida, dependiendo de sus contextos. De este modo, la noción del curso de vida aporta mucho para comprender la juventud asociada a los cambios en las trayectorias educativas y a las transiciones que ocurren durante esta etapa (Pallas, 2003), como es el caso de la transición postsecundaria, en la que se identifican diferentes márgenes de posibilidad y limitación para que los jóvenes puedan ejecutar sus proyectos de vida. Este marco conceptual resulta útil sobre todo para abordar contextos rurales muy poco estudiados y donde es reciente la expansión de la educación superior.

\section{Avances educativos: Algunas cifras estadísticas}

Así como ocurre en los niveles previos, la culminación de la educación secundaria en el Perú es creciente y esto es significativo en muchas zonas rurales del país. Específicamente, en la región Apurímac, donde se desarrolla la investigación, encontramos que la tasa de conclusión de la secundaria del grupo de edad de 17 a 19 años presenta un 66,5\% en el año 2012 y un 71,8\% en el año 2015 (Escale-Minedu, 2015). Asimismo, la tasa de transición a la educación superior en esta región en los últimos 4 años (2012-2015) muestra un crecimiento de 6,4\% (Escale-Minedu, 2015). Aunque no tenemos datos desagregados por provincias, suponemos que la población de egresados de la secundaria está generando una transformación en el espacio local.

Este significativo avance educativo en las recientes generaciones se relaciona también con un contexto de mayor oferta y demanda en la educación superior, tanto técnica como universitaria ${ }^{2}$. Al respecto, los hallazgos del segundo censo universitario (Cenaun-Inei, 2010) nos ofrecen interesantes pistas para comprender el panorama actual de la educación superior en áreas rurales. A nivel nacional, encontramos un crecimiento poblacional anual en las universidades privadas, con un $9,3 \%$, frente a las universidades públicas, con un $3,2 \%$. Además, en 2010, fue mayor la población que ingresó a las universidades privadas (37,1\%) que a las públicas $(28,4 \%)$ al año siguiente de culminar la secundaria. En el caso de las regiones al interior del país, también, se registra esta tendencia: un 37\% estudia en universidades privadas; y un $34,1 \%$, en universidades públicas.

Esta tendencia de mayor ingreso a universidades privadas también se relaciona con un modelo más flexible de ingreso en comparación con los requisitos de ingreso a las universidades públicas. Sobre ello, el censo evidencia que la

2. Tal como señala (Díaz, 2008) se registra una mayor demanda y oferta en la educación superior universitaria y técnica. En cuanto a la educación universitaria, se evidencia un mayor crecimiento poblacional en universidades privadas, sobre todo, a partir de mediados de los 90, que también corresponde a un contexto de liberación del mercado educativo. Por el contrario, en las universidades públicas, se evidencia un estancamiento en el crecimiento poblacional. Por su parte, en la educación técnica, se registra un mayor incremento poblacional en los institutos tecnológicos privados. 
preparación universitaria es un requisito para la mayoría de postulantes a la universidad pública, lo que no sucede en la universidad privada (Cenaun-Inei, 2010). Asimismo, la preparación preuniversitaria ha ido decreciendo en los últimos años. Mientras que en 1996 se puede identificar un 50,8\% de estudiantes universitarios que se habían preparado en academias, para 2010, notamos que solo un 30,7\% contaba con dicha preparación. Entre otros aspectos relevantes, encontramos que el principal motivo para elegir una universidad es su prestigio (54\%), sin diferenciar si se trata de una institución pública o privada (Cenaun-Inei, 2010).

En este contexto, en la región Apurímac, se observa una tasa de crecimiento universitario de 12,4\% anual entre el período 1996-2010. Este crecimiento la posiciona como la segunda región con mayor población universitaria (10.134 estudiantes en el año 2010). En cuanto a la composición de estudiantes universitarios, encontramos que la expansión de la oferta universitaria ha generado una mayor permanencia de jóvenes que culminan la secundaria en la región. Así, mientras que en 1996 un 56\% de estudiantes estudiaba fuera de la región por falta de oportunidades educativas locales, en 2010, solo un 9,8\% estudió fuera de ella.

En líneas generales, la mayor permanencia y culminación de la educación secundaria, y la expansión en la oferta y demanda de la educación superior -como muestran las cifras- son dos fenómenos que bien pueden responder principalmente a cuatro factores: las presiones demográficas, un proceso de urbanización del país, la rentabilidad de la educación en el mercado laboral y las expectativas de movilidad social (Díaz, 2008). A la luz de estos factores, las recientes generaciones están afrontando cambios en varias regiones del país y precisamente Apurímac constituye una de las regiones que destaca por su crecimiento educativo en la última década. En relación con la educación universitaria, solo hasta el año 2012 se registraron 3 universidades públicas y 2 privadas $^{3}$, mientras que, en el caso de la educación no universitaria, actualmente, se registran 17 institutos tecnológicos en la región (Alemán, 2015). Como veremos, esta expansión de la oferta en la educación superior atrae a los crecientes egresados de la secundaria, quienes independientemente del nivel educativo de sus familiares buscan ser profesionales en sus localidades.

\section{El curso de vida y la juventud}

Más allá de la data estadística presentada, este estudio se sitúa dentro del enfoque de curso de vida ${ }^{4}$, desde el cual se comprende a la juventud como una

3. Tal es el período en el cual se desarrolló el trabajo de campo.

4. El curso de vida es un enfoque teórico y metodológico que aparece en la década de los 70; este destaca el papel en la acción de los individuos dentro de márgenes estructurales y sus posibilidades como limitaciones cambiantes a lo largo del tiempo (Elder, 1994). 
etapa diversa de tránsito a la adultez (Juárez \& Gayet, 2014). Se trata de un enfoque teórico-metodológico que integra la dimensión estructural con las posibilidades de agencia que tienen los sujetos (Elder, 1994; Ulrich, 2004; Heinz \& Krüger, 2001). De este enfoque, es importante retomar dos conceptos clave: las trayectorias y las transiciones. Las trayectorias se entienden como líneas de largo plazo en la vida de la persona, mientras que las transiciones son aquellos cambios de estado, posición o situación que dan forma a la trayectoria (Blanco, 2011; Abbott, 1997; Pallas, 2003). En este sentido, desde el curso de vida, la juventud se asume como una etapa de transición a la vida adulta que es sociohistórica y diversa (Sepúlveda, 2013), y que, debido a los contextos actuales de acelerados cambios especialmente en el mercado de trabajo y la educación, involucra distintos tipos de trayectorias y muchas veces con procesos inciertos (Dávila 2005; Juárez \& Gayet, 2014).

De esta forma, ser joven también se relaciona con la experiencia ${ }^{5}$ en espacios de socialización, donde las dimensiones educativa, laboral y familiar ocupan un rol central en la vida de los jóvenes. Para el caso de la experiencia de ser joven en ámbitos rurales, los aportes del estudio de Olivera (2010) señalan la importancia de la organización social, que moldea los modos de socialización de estos actores sociales. Para el caso de la socialización escolar, la convivencia en la escuela, además de ser un espacio de aprendizaje en valores orientados a mejorar la inserción social en el futuro, posibilita la interacción entre varones y mujeres, hecho que fuera de este contexto no es posible debido a ciertos factores de exclusión que limitan la interacción entre ambos géneros ${ }^{6}$. Esto, a su vez, se relaciona con la organización familiar, en la que el trabajo se convierte en un espacio de aprendizaje diferenciado para los varones y las mujeres (Olivera, 2009). Al respecto, importa mucho mirar la situación de los jóvenes rurales en el marco de la experiencia escolar y en los niveles de participación en el mundo del trabajo, que se desarrolla desde temprana edad, porque ello influye en sus tránsitos postsecundarios (Ames, 2013).

Con ello, también, intento resaltar que las experiencias de los jóvenes rurales no pueden deslindarse de los intereses familiares, y que la socialización desarrollada en la escuela y el mundo laboral es parte de acuerdos continuos

5. La experiencia en la perspectiva de Dubet (2010 ) se entiende como una integración de las lógicas de acción: i) lógica de integración a la sociedad, ii) una lógica estratégica en un espacio competitivo y iii) lógica de subjetivación, en la que aparece la capacidad crítica y de interpretación. En este sentido, en la experiencia escolar, los jóvenes son sujetos que se integran a la sociedad desarrollan estrategias para alcanzar sus metas propuestas y buscan adquirir una autonomía manteniéndose dependientes de los adultos (Dubet \& Martuccelli, 1997).

6. Como parte de los factores de exclusión, se señalan las limitaciones para independizarse e insertarse en el mundo laboral en el caso de los varones, mientras que, en el caso de las mujeres, el control sobre su sexualidad limita sus posibilidades de estudio y trabajo (Olivera, 2009). 
con la familia a lo largo del tiempo. Aquí se retoma el enfoque de interdependencia negociada (Punch, 2002), que ayuda a comprender la naturaleza de la organización familiar campesina; en esta, el colectivo familiar integrado tiene objetivos comunes y, en función de ello, organiza a todos sus miembros para lograrlo. En esta lógica de producción y reproducción de las familias campesinas, en la cual la educación tiene un papel central, la interdependencia negociada es la suma de acuerdos y compromisos entre padres e hijos a fin de alcanzar beneficios comunes.

Con estas aproximaciones, comprendo a los jóvenes de este estudio como a una población que se encuentra entre los dieciséis y veinte años, que culmina la educación secundaria desarrollando un proyecto de vida $^{7}$ orientado a la educación superior y con el cual afrontan su transición postsecundaria (Dávila, 2005). Dado que la juventud se entiende también como una transición a la vida adulta, el período postsecundario no es solo un tiempo en el cual se desarrollan mayores esfuerzos para alcanzar el objetivo del proyecto de vida, sino que también involucra el deseo por ampliar los niveles de autonomía frente a las generaciones mayores. Las dimensiones educativas, familiares y laborales en este momento específico generan cambios que pueden alterar el rumbo de las trayectorias. Para comprender las transiciones de los jóvenes, me apoyo también en estudios empíricos -como el de Johnson-Hanks (2002) en relación con las coyunturas vitales ${ }^{8}$, y el de Thomson et al. (2002) sobre los momentos críticos $^{9}$ - para señalar que los cambios que afrontan los jóvenes en este período también afectan sus posibilidades de decisión y desarrollo de niveles de autonomía.

Las transiciones postsecundarias transforman el curso de vida de los jóvenes, sobre todo, en contextos de alta valoración de la educación superior y en familias en las que no existen profesionales ${ }^{10}$. Así, al ser la educación superior un proyecto de vida y un proyecto educativo familiar, se elaboran expectativas alrededor de la misma, las cuales, como veremos, pueden llegar a concretarse, mientras que otras, no necesariamente. Todo dependerá de las situaciones

7. El proyecto de vida se entiende como un modelo anticipatorio que organiza las actividades y el comportamiento del individuo, que le permite regular sus expectativas y diseñar posibles estrategias (Hernández, 2006). Parte de una construcción paulatina a lo largo de la experiencia.

8. El curso de vida está compuesto por coyunturas vitales; aquellos momentos en los que se pueden tomar diversos caminos (producto de decisiones y posibilidades) son también potencialidades que se piensa definirán la vida de la persona.

9. Los momentos críticos son aquellos momentos clave que originan cambios en la persona y que están interconectando los niveles de agencia frente a márgenes estructurales.

10. Esto se relaciona con familias no educadas, pero que tienen una alta valoración educativa porque reconocen a la educación como vehículo de movilidad social (Benavides, Olivera \& Mena, 2006). 
que se presenten, que pueden facilitar o dificultar las oportunidades tanto de varones como de mujeres.

Entre los estudios previos en relación con el tema propuesto, se destacan algunos, como el de León y Sugimaru (2013), quienes identifican los factores asociados a la toma de decisiones de los jóvenes. Si bien todos tienen la expectativa de cursar los estudios superiores, las estrategias empleadas a la salida de la secundaria son distintas (estudiar, trabajar o alternar estudio y trabajo al mismo tiempo), y pueden potenciar o limitar las posibilidades de acceder a los estudios superiores. Los hallazgos muestran, entonces, que las transiciones juveniles en este período no son secuencias lineales (Otero, 2010) y que dependen de condiciones previas en la socialización. De igual modo, un estudio de Guerrero (2013) sobre el factor familiar y la experiencia escolar que alimentan la intención de los jóvenes para postular a la universidad destaca la importancia de las características educativas familiares, del rendimiento escolar en áreas de matemática y comprensión lectora, y del apoyo institucional de la escuela con talleres de orientación vocacional, simulacros de examen, entre otros. Finalmente, un estudio reciente de Cotler (2015) sobre estudiantes en el programa Beca 18 muestra que dicha beca permite que los jóvenes de los sectores más pobres y de zonas rurales que culminan la secundaria con un rendimiento superior al promedio puedan acceder a la educación superior. Sin embargo, como vemos en otro estudio, el tránsito a la educación superior es un proceso complejo que también puede interrumpirse debido a que se encuentran problemas de tipo vocacional, académico, económico, de salud, etc. (Aramburú, 2014).

Retomando aportes de los mencionados estudios, este trabajo tomará en cuenta el proyecto de vida (Hernández, 2006), en el que expectativas y estrategias moldean la experiencia. Las expectativas puestas en la educación superior implicaron considerar tres tipos de factores: i) económicos, en lo que respecta los costos directos de inversión; ii) educativos, los cuales se relacionan con los aprendizajes escolares; y iii) culturales, en relación con las valoraciones sobre el prestigio de las instituciones privadas y públicas. Estas expectativas, a su vez, motivan el diseño de estrategias como acciones posibles (Dubet, 2010), que, como veremos, se van readaptando a medida que cambian las expectativas iniciales.

En este sentido, este estudio contribuye a rellenar el vacío de información que tenemos sobre jóvenes en contextos rurales. Sabemos que, en la última década, se han expandido las oportunidades educativas en varias regiones que también son focos comerciales importantes al interior del país (Pérez, 2008). Sin embargo, si bien ahora parece ser más viable que las nuevas generaciones accedan prontamente a la educación superior, sus transiciones también pueden ser muy frágiles. Esto se debe, por ejemplo, a que no existen referentes familiares cercanos que hayan cursado la educación superior, a que existe una mayor presión para alcanzar logros en el corto plazo, a que los jóvenes pueden no tener los acompañamientos necesarios en el proceso, a que pueden verse 
limitados a tomar decisiones o a que sienten que la secundaria no les brindó las suficientes herramientas para acceder a la universidad.

\section{Metodología y contexto de estudio}

Para fines del estudio, se retoma el enfoque del curso de vida como metodología que emplea las historias de vida para analizar las trayectorias y las transiciones (Blanco, 2011). Para ello, se realizó un trabajo de campo etnográfico a lo largo de tres meses, en los cuales, desde un enfoque cualitativo, se realizaron entrevistas semiestructuradas y observaciones (Maxwell, 2005; Guber, 2001). Si bien los actores centrales eran los jóvenes egresados, debemos resaltar que sus transiciones ocurren en el marco de su interacción con diversos actores en espacios interconectados. Por ello, se entrevistó a los padres de familia, y a algunos docentes y jóvenes que no pertenecieron a la muestra. Cada uno de ellos brindó información relevante que fue contrastada con la observación participante para triangular la información obtenida.

El trabajo de campo se realizó durante los meses de agosto a octubre del año 2012, e incluyó una visita durante el mes de abril de 2013. La muestra fue tomada a partir de la lista de egresados de quinto año del colegio seleccionado $^{11}$, que, por ese entonces, contaba con cuatro listas correspondientes a las cuatro secciones de quinto de secundaria. De esta lista, se identificaron a aquellos jóvenes que provenían de las comunidades y de los barrios cercanos al distrito, y que fuesen tanto varones como mujeres. Luego de ello, recibí el apoyo de un docente del colegio para obtener una información inicial sobre la ubicación de algunos de los jóvenes, quienes luego ayudaron a ubicar a sus excompañeros de colegio.

Según los dos criterios establecidos (lugar de procedencia y género), se seleccionó a un grupo de veinte jóvenes: once varones y nueve mujeres. Diez de ellos residían en la parte más urbana y otros diez en sus comunidades. El grupo seleccionado se encontraba por aquel entonces entre los dieciséis y veinte años de edad. A cada uno de los jóvenes se le aplicó una guía de entrevista semiestructurada y se conversó sobre su historia de vida. La recopilación de información de los casos se situó en dos tiempos: el tiempo presente (fuera de la secundaria) y el tiempo pasado en relación con la experiencia de la secundaria. Esta recopilación de información también involucró observaciones durante algunos acompañamientos que se hicieron a los jóvenes. Posteriormente, se aplicó una entrevista semiestructurada a varios de los padres de familia y se tuvieron conversaciones abiertas con otros jóvenes que, por ese entonces, se encontraban en quinto de secundaria en el mismo colegio, y quienes, en algunos casos, eran familiares cercanos de los jóvenes de la muestra seleccionada.

11. La selección del colegio fue posible gracias a que se establecieron contactos previos desde Lima y porque este es un colegio histórico que alberga a mayor población rural que viene de las comunidades. 


\subsection{Contexto de estudio}

La investigación se desarrolló en el distrito de San Jerónimo, que se encuentra a unos $2900 \mathrm{msnm}$ y a $2 \mathrm{~km}$ de la capital provincial de Andahuaylas. Un aspecto resaltante es que San Jerónimo forma parte de un valle altamente productivo, que abastece no solo a la población de la región, sino también a otras regiones como Cusco y Ayacucho. Como colinda con Andahuaylas, se tiene acceso a servicios básicos y al desarrollo comercial de diversos productos principalmente agrícolas. Sin embargo, también, existe una interconexión entre el área urbana (los barrios cercanos a las plazas) y las comunidades rurales, lo cual facilita la movilidad de la población del campo a la ciudad y viceversa por motivos comerciales y educativos.

Con respecto a la oferta educativa, hallamos diversas escuelas públicas y privadas en el nivel primario y secundario ${ }^{12}$. En cuanto a la educación superior, la expansión es más reciente y abarca diversos institutos técnicos y universidades públicas y privadas. Entre los institutos más resaltantes, se encuentran el Senati, la escuela Todas las Artes y el Instituto Pedagógico Superior de Andahuaylas (Isspa) ${ }^{13}$. En el caso de la educación universitaria, hallamos dos universidades públicas en Andahuaylas ${ }^{14}$, tales como la Universidad Nacional José María Arguedas ${ }^{15}$ (creada en el año 2004) y la filial de la Universidad Nacional San Antonio Abad del Cusco ${ }^{16}$. En lo que respecta al ámbito privado, están la Universidad Tecnológica los Andes ${ }^{17}$ (Utea) y la Universidad Alas Peruanas $^{18}$ (Uap). La mayor presencia de universidades ha generado la aparición de centros preuniversitarios de los mismos centros de estudio, como ocurre con la universidad José M. Arguedas. Asimismo, se han creado nuevas academias (como la Aduni, Cesar Vallejo, Trilce y Pitágoras), que cuentan con distintos programas de preparación dependiendo de la universidad a la cual se piensa

12. En cuanto a instituciones educativas pioneras, en el distrito de San Jerónimo, se encuentra el colegio más antiguo de la región, donde estudiaron muchos de los docentes que actualmente trabajan ahí.

13. Este instituto fue hasta aproximadamente los años 90 la única opción en la educación superior.

14. En la región, existen tres universidades públicas; de ellas, solo la Universidad Micaela Bastidas tiene su sede en la capital de la región.

15. Se ofrecen tres especialidades: Administración, Ingeniería de sistemas e Ingeniería Agroindustrial.

16. Se ofrecen dos especialidades: Ingeniería Agropecuaria y Obstetricia.

17. Se ofrecen seis especialidades: Contabilidad, Agronomía, Derecho, Educación, Enfermería e Ingeniería Ambiental.

18. Se ofrecen doce especialidades: Estomatología, Obstetricia, Psicología, Administración, Finanzas, Turismo, Gastronomía, Derecho y Ciencias políticas, Ingeniería Civil, Ingeniería ambiental, Arquitectura. 
postular. Finalmente, cabe anotar que, durante el trabajo de campo, se registró la reciente llegada del programa Beca 18 y una campaña para la convocatoria del programa Jóvenes a la Obra.

A partir de ello, vemos que el contexto ofrece diversas oportunidades. Las generaciones jóvenes reconocen que tienen mayores posibilidades de acceder a los estudios superiores y ello trae consigo mayores expectativas en torno a sus preocupaciones sobre el futuro. A continuación, se expone, de manera resumida, las trayectorias de cinco jóvenes que formaron parte de la muestra, en las que se destacan las acciones y actitudes que toman y cambian en el proceso.

\section{Los jóvenes: características previas}

Si bien los tránsitos postsecundarios muestran cambios, se desarrollan a partir de la experiencia previa en espacios de socialización centrales, como son la familia y la escuela. Para empezar, es fundamental comprender la socialización temprana de la familia con el mundo del trabajo, que también se relaciona con los modos de producción y reproducción familiar, y el rol que cumplen los jóvenes en este espacio (Punch, 2002; Olivera, 2009).

La pluriactividad en los espacios rurales ayuda a comprender lo que ocurre con las dinámicas familiares (Pérez, 2008). Aunque los jóvenes provienen de hogares que se dedican principalmente a la agricultura y el comercio de sus productos, también, deben complementar sus ingresos con periodos de actividad en otros oficios, tales como construcción, seguridad, ventas, entre otros. En este contexto, la zona de residencia desempeña un papel fundamental para la reproducción familiar. Como veremos, los cinco casos expuestos residen en diferentes lugares: algunos provienen de la parte más urbana de San Jerónimo; y otros, de la parte rural en las comunidades.

Tabla 1. Lugares de residencia

\begin{tabular}{cl}
\hline Jóvenes & \multicolumn{1}{c}{ Lugares de residencia } \\
Mariana & Barrio cercano a la plaza de San Jerónimo \\
Nadia & Chulcuiza (Comunidad) \\
Miguel & Champaccocha (Comunidad) \\
Marisol & Poltoccsa (Comunidad) \\
Fabián & \\
\hline
\end{tabular}

Fuente: Elaboración propia

El área rural y la urbana están claramente interconectadas. Esta interconexión ayuda a la movilidad de las familias y permite comprender las posibilidades que tuvieron los jóvenes que residen en las comunidades para estudiar en la parte más urbana. Muchas familias de la zona rural emplean una serie de 
estrategias para facilitar una educación más urbana para los hijos. Como estrategias, encontramos i) vivir en la casa familiar que fue producto de la compra de un terreno en la ciudad, ii) vivir en un cuarto alquilado o en la casa de un familiar, y iii) vivir en una casa hogar ${ }^{19}$. Resulta importante detenernos a considerar este aspecto, dado que forma parte de las estrategias de doble residencia (De la Cadena, 1988) que utilizan las familias para facilitar la pluriactividad comercial y, sobre todo, la educación de los hijos.

A partir de la experiencia de estudiar en la ciudad y de estas tres estrategias, los jóvenes asumen una serie de responsabilidades con sus familias. Conceptos como «ayuda» y «trabajo» permiten comprender cómo se organizan las responsabilidades, y, además, cómo se construye un sentido de responsabilidad y progresiva autonomía. Para los jóvenes de este estudio, la educación es igual de importante que las responsabilidades con el trabajo, aunque los modos y tiempos dedicados a trabajar sean diversos. Los jóvenes han desarrollado una consciencia de participación necesaria y voluntaria a lo largo del tiempo, pese a que establecen una distinción entre el trabajo y la ayuda. Trabajo se considera como toda actividad dirigida al beneficio de terceros, de los cuales se recibe un pago llamado jornal; por el contrario, se denomina ayuda a aquellas actividades que son parte de las responsabilidades con la familia nuclear, incluso extensa, por las que no se recibe un pago monetario. De esta manera, los jóvenes trabajan y ayudan organizando tiempos para no abandonar su vida escolar.

Un hecho importante sobre la trayectoria ocurre durante las vacaciones escolares, puesto que es frecuente que los jóvenes salgan a emplearse en la ciudad para trabajar en ventas, construcción, cuidado de niños y limpieza. También, está el trabajo y la ayuda brindada en la chacra, sobre todo, durante los tiempos de siembra y cosecha. En este caso, los varones se diferencian de las mujeres, porque disponen de mayores libertades para conseguir un trabajo que les permita distanciarse del hogar por algunos meses. A diferencia de ellos, son pocas las mujeres que trabajan fuera del hogar debido a que es mayor el grado de control por parte de sus padres.

Todo lo mencionado también corrobora lo ya mencionado en estudios previos, como los de Ames (2013) sobre la participación y responsabilidad de los hijos a lo largo del tiempo, así como el estudio de Olivera (2009), en el que se destaca los modos de participación de los jóvenes de acuerdo con la organización social. Lo central aquí es mencionar que los modos de participación a lo largo del tiempo repercuten en las posibilidades de desarrollo de autonomía que tienen los jóvenes al momento de conducirlos en sus tránsitos postsecundarios.

19. Existe una casa hogar que acoge a adolescentes varones que provienen de las comunidades solo mientras estudian la secundaria en los colegios de la ciudad. Este albergue está destinado a adolescentes de escasos recursos. 


\section{La transición postsecundaria}

Poco antes de culminar la secundaria, surgen una serie de preguntas y dudas respecto del proyecto de vida. Los jóvenes se preparan para salir de la secundaria teniendo expectativas y estrategias más o menos claras. Las expectativas puestas en la educación superior muestran la intención de prolongar las trayectorias educativas, a la vez que se posterga la idea de formar un hogar propio (Otero, 2010).

Durante el último año de la secundaria, los estudiantes recibieron charlas de orientación vocacional en los espacios de tutoría. Los jóvenes consideraron importante recibir orientaciones, pues pudieron complementar las conversaciones que tuvieron en sus hogares y con sus amigos fuera de la escuela. Esto les brindó las herramientas iniciales para pensar en posibles estrategias que debían realizar, aunque, como en todo proyecto de vida (Hernández, 2006), se señala que el diseño de estrategias no concluye al finalizar la secundaria, sino que sienta las bases que luego serán reinterpretadas en la transición postsecundaria.

Reconstruyendo las historias, encontramos que, durante los últimos meses en la secundaria, los jóvenes se encontraban medianamente preocupados por qué carrera elegir, en qué academia prepararse y cómo costearían sus estudios. Frente a ello, el colegio brindó importante información mediante las visitas que recibieron de algunas universidades (principalmente, de la Universidad Alas Peruanas) e, incluso, de algunas academias. Por su parte, los profesores se mostraron interesados en aconsejar a sus estudiantes sobre qué podrían estudiar. Por otro lado, las familias, específicamente los padres, consultaron a los hijos sobre su interés en seguir estudiando, puesto que buscaban ayudar a quienes realmente quisieran continuar con los estudios. En esta fase, convergen momentos de entusiasmo y preocupación en los jóvenes.

A partir de estos diversos estímulos, los jóvenes identificaron distintos aspectos que consideraron clave en la formulación de sus expectativas y sus posibles estrategias. Hemos agrupado la información obtenida en tres factores específicos que acompañan la transición postsecundaria. A continuación, exponemos cada uno de ellos: 
Tabla 2. Factores involucrados en la formulación de expectativas

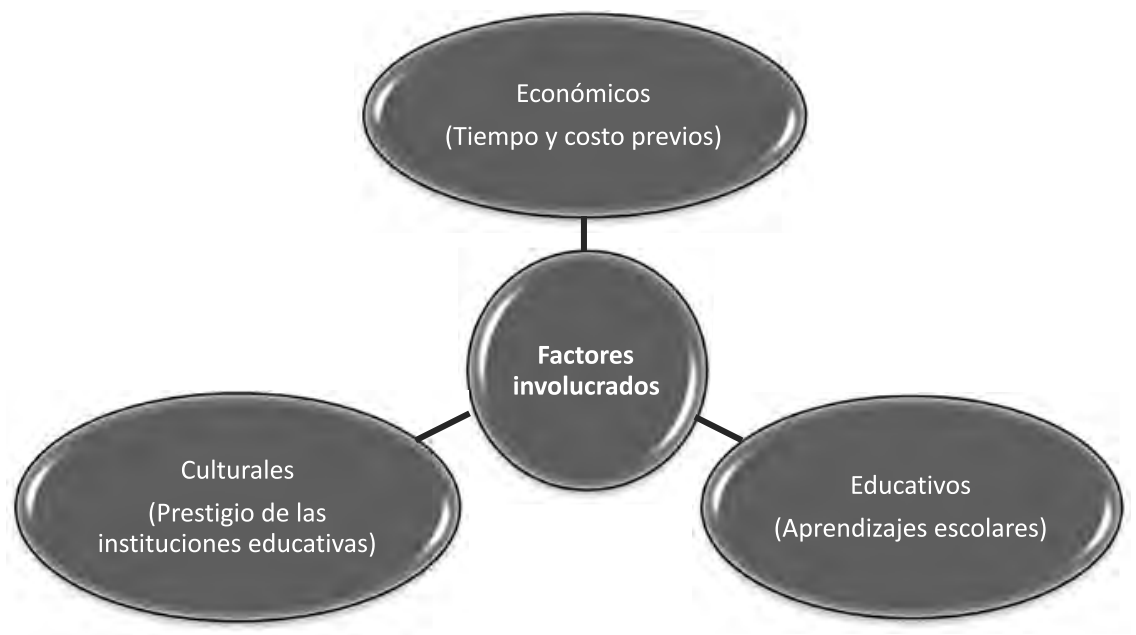

Fuente: Elaboración propia

En el gráfico, se exponen tres factores específicos interconectados:

- Los factores económicos involucran costos monetarios principalmente en función del tiempo de preparación antes de acceder a la educación superior. En este caso, es central el tiempo y costo invertido en la preparación en la academia, así como el costo de postulación a la universidad, sin considerar otros gastos indirectos, como transporte y alimentación.

- Los factores culturales hacen referencia a las percepciones sobre lo que tiene un mayor reconocimiento social en la educación superior. En relación con ello, la universidad tiene un mayor prestigio sobre los institutos técnicos, debido a que la educación universitaria se considera como un estudio más completo y mejor reconocido por el mercado laboral (Díaz, 2008). Sin embargo, entre las universidades públicas y privadas, encontramos que las públicas son las que tienen un mayor reconocimiento, porque suponen un mayor esfuerzo académico para ingresar ${ }^{20}$.

- Los factores educativos refieren a todos aquellos aprendizajes propiamente escolares. Los jóvenes consideran su rendimiento académico en áreas de Matemática y Letras al momento de elegir una carrera (Guerrero, 2013). Dependiendo de los casos, algunos jóvenes indican tener un mayor dominio en los cursos de Letras que en los cursos de Ciencias y viceversa. Esto les ayuda a identificar opciones más viables en las cuales invertir.

20. Además, prefieren estas instituciones, porque son las más cercanas a sus posibilidades en términos económicos. 
La combinación de estos tres factores ayuda a formular las expectativas y diseñar estrategias de manera más concreta. Sin embargo, en este proceso, cobra especial importancia el rol que asumirán las familias luego de que los jóvenes concluyan la secundaria. Así, la formulación de expectativas y estrategias desde el final de la secundaria en adelante se entiende también como una interdependencia negociada (Punch, 2002) y arreglos familiares (Olivera, 2010). Ahora bien, estos compromisos y modos de apoyo se diferencian en cada caso, pese a que, en general, se trate de familias (padres e hijos) que le brindan un alto valor a la educación (Benavides, Olivera y Mena, 2006). De lo observado, podemos compartir dos conclusiones: i) el apoyo familiar no solo se traduce en apoyo económico y ii) recibir menor apoyo económico de la familia no siempre condiciona a los jóvenes a abandonar sus expectativas educativas.

Una vez que los jóvenes egresaron de la secundaria, se pudo ser testigo de cuáles fueron las estrategias que venían realizando y también los planes que habían modificado. Mediante las estrategias empleadas, se identifica un elemento central que ocurre durante esta transición: las seguridades y la incertidumbre que acompañan este período. Vale mencionar que la incertidumbre se entiende como ajena a la voluntad y que es característica a lo largo del curso de vida de la persona (Elder, 1994). Al respecto, proponemos una tabla en la que ubicamos los cinco casos tomados de la muestra. Cada caso se encuentra en un cuadrante distinto, en el que se comparan los niveles de seguridad en relación con los niveles de apoyo económico.

Tabla 3. Niveles de seguridad mediados por el nivel de apoyo económico familiar

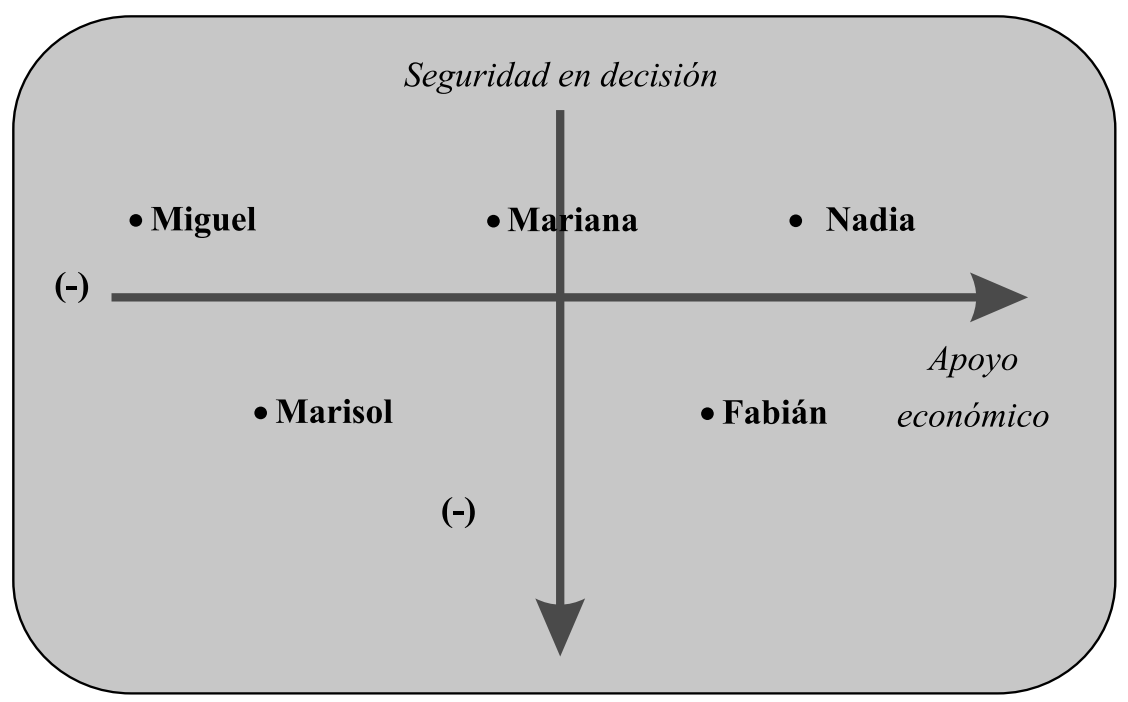

Fuente: Elaboración propia 
Los cinco casos representan situaciones diferentes sobre niveles de seguridad y niveles de apoyo económico recibido ${ }^{21}$, con lo cual se resalta que el apoyo económico familiar no siempre condiciona las posibilidades o limita las transiciones de los jóvenes. En el primer caso, Miguel, representa al grupo de jóvenes que muestra un mayor nivel de seguridad sobre sus expectativas educativas, a pesar de reconocer que su familia no podría ofrecerle el apoyo económico necesario para llevarlas a cabo ${ }^{22}$. El segundo caso, Mariana, encarna a aquellas jóvenes que tienen mayor seguridad en sus expectativas y cuentan con cierto respaldo económico familiar ${ }^{23}$. El tercer caso, Nadia, representa a aquellos jóvenes que tienen una mayor seguridad en sus expectativas y cuentan con el apoyo económico familiar necesario ${ }^{24}$. El cuarto caso, Marisol, figura a los jóvenes que sienten una mayor incertidumbre por el futuro y no cuentan con el apoyo económico familiar suficiente ${ }^{25}$. En el último caso, Fabián representa a aquellos que cuentan con apoyo económico familiar y tienen alta valoración educativa, pero que no se sienten seguros sobre qué estudiar en el corto plazo $y$, por ello, salen a buscar otras oportunidades, incluso fuera de la provincia ${ }^{26}$.

Sobre lo descrito, a continuación, se exponen las transiciones postsecundarias de estos cinco jóvenes a fin de mostrar cuáles fueron sus actitudes frente a las oportunidades y dificultades encontradas tras finalizar la secundaria.

\subsection{Miguel: mayores seguridades frente a la escasez de apoyo económico familiar}

Miguel es el menor de seis hermanos, de los cuales solo dos lograron terminar la secundaria, e hijo de padres que no lograron culminar la primaria. Proviene de una familia de escasos recursos, en la cual el padre y la madre se dedican a las actividades agrícolas. Su trayectoria educativa ha estado acompañada por diversas actividades laborales, temporales y continuas, a partir del tercer año de secundaria. Señala que desde muy pequeño ayudó a sus padres en la chacra, como lo hacían sus hermanos, y con el paso de los años comenzó a trabajar por jornales como peón. Con su trabajo, logró costearse algunos gastos de escolaridad y aprendió a tomar mayores responsabilidades en el hogar. Sus padres acompañaron su escolaridad mediante incentivos morales sobre el valor

21. Esto es enteramente interpretación personal a partir de las entrevistas realizadas.

22. Este grupo originalmente estaba conformado por cuatro jóvenes varones.

23. Este grupo originalmente estaba compuesto por cinco jóvenes: cuatro mujeres y un varón.

24. Este grupo originalmente estaba conformado por dos varones y dos mujeres. Un dato adicional de este caso es que los jóvenes tienen hermanos mayores que estaban cursando sus estudios superiores.

25. Este grupo estaba integrado por cinco jóvenes: dos mujeres y tres varones.

26. Este grupo lo constituían dos jóvenes: un varón y una mujer. 
educativo, al igual que sus hermanos mayores, quienes le decían que debía terminar el colegio y aspirar a convertirse en un profesional.

En quinto de secundaria, Miguel elaboró un proyecto de vida que, hasta hace un año atrás, no lo había considerado como un hecho prioritario. Este consistía en estudiar Agroindustria en la Universidad José María Arguedas. Este plan surgió a partir de tres hechos: i) encontró interés en un curso sobre formación laboral, en el que la profesora encargada promovía que los alumnos utilizaran los aprendizajes como una opción de carrera profesional; ii) recibió orientación en el colegio sobre la oferta educativa local y las posibilidades profesionales; y iii) tuvo conversaciones casuales con familiares que preguntaban qué carrera pensaba estudiar para cuando terminara la secundaria. El resultado de estos tres hechos lo ayudó a tomar la decisión de estudiar Agroindustrias en una universidad. Sin embargo, reconocía que debía prepararse previamente en la academia, para lo cual necesitaría dinero adicional, puesto que no contaba con el apoyo económico de sus padres debido a los escasos recursos de su familia. Lo importante es que, pese a sus circunstancias aparentemente desfavorables, Miguel nunca abandonó sus expectativas: «siempre hay la falta de dinero, siempre la economía, pues, por eso, me he ido a trabajar dos meses... No me he ido a preparar en marzo, en vacaciones de verano, sino en junio ya» (Miguel, 17).

Gráfico 1. Secuencia de eventos en la transición de Miguel

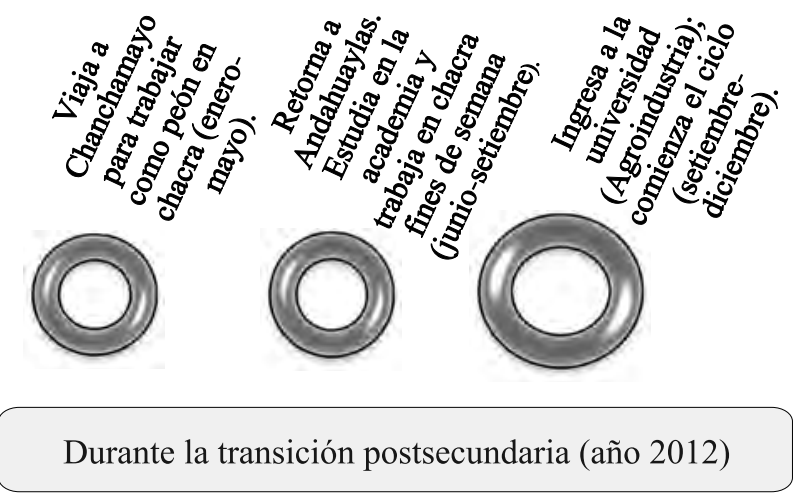

Fuente: Elaboración propia

Durante varios meses luego de culminar la secundaria, Miguel trabajó para ahorrar dinero y logró matricularse en la academia para postular a la carrera de Agroindustrias. De manera paralela a su preparación, los fines de semana trabajaba en la chacra, ya sea ayudando a sus padres o como peón por jornal. Como comentaba, pese a que su familia no podía apoyarlo económicamente con los gastos de preparación, siempre vieron la forma de organizarse para ayudarlo durante este tiempo. En este caso el tiempo de preparación en la 
academia fue corto, ya que Miguel, quien tenía un buen rendimiento escolar, ingresó a la universidad pocos meses después. Siempre tuvo seguridad respecto a lo que quería estudiar y fue clave esforzarse trabajando y estudiando independientemente de lo que su familia pudiese ofrecerle. Ello no significa que sus padres no se preocupaban por su bienestar, sino que él tenía mayor autonomía para tomar decisiones y su familia lo apoyaba acompañándolo moralmente.

\subsection{Mariana: mayores seguridades y necesidad del apoyo económico familiar para alcanzar los objetivos educativos}

Mariana vive en uno de los barrios de San Jerónimo, con su madre y sus cuatro hermanos menores. Su madre no logró culminar la secundaria y, actualmente, sus hermanos se encuentran en la etapa escolar. Cada uno de los miembros del hogar asume distintas responsabilidades en los quehaceres de la casa, apoyo en la chacra y cuidado de los animales. Al igual que sus hermanos menores, Mariana colabora en distintas tareas. Sin embargo, por ser la hermana mayor, muchas veces ha asumido los cuidados de sus hermanas menores, debido a que su madre salía a trabajar en el comedor de una universidad cercana. Además de ello, durante las vacaciones del último año de secundaria, estuvo trabajando unos meses como cuidadora de un niño en casa.

Desde tercero de secundaria, Mariana deseaba ser policía. Este interés surge a partir de escuchar comentarios de amistades sobre los beneficios económicos que tendría un policía en servicio y, sobre todo, por el corto tiempo (un año) que toma la preparación. Al llegar a quinto de secundaria, Mariana se sintió segura sobre lo que quería estudiar cuando acabase el colegio, pero, con el paso de los meses, se desanimó de postular a la escuela de policías, porque se dio cuenta de que no tenía la edad requerida (18 años) y empezó a desarrollar un mayor interés por la universidad.

El mayor interés por la universidad surge a mediados del último año escolar, cuando recibe información sobre la oferta educativa local. Quería ir a la universidad, debido a que consideraba que era más prestigioso que estudiar en un instituto, aunque no estaba segura sobre qué carrera quería elegir. No quería estudiar Ingeniería, ya que sabía que en el examen de admisión le pedirían un mayor puntaje en el área de números. Por otro lado, reconocía que solo podía estudiar en una universidad pública, por lo cual recibiría un mayor reconocimiento a su esfuerzo. Además, sabía que debía prepararse en la academia y, considerando sus opciones, decidió postular a la carrera de Administración. «Lo importante era no perder el tiempo y mejor estudiar una carrera en la universidad mientras tanto que pueda tener 18 y luego poder postular a la policía» (Mariana, 16).

El plan de Mariana para los siguientes años implicó cuatro acciones: i) sentirse segura sobre lo que quería estudiar en la universidad; ii) postergar la postulación a la escuela de policía por no tener la edad requerida para postular, 58 I pero con la idea de llegar a ser policía más adelante; iii) tomar mayor responsa- 
bilidad con sus estudios; y iv) acordar con su madre buscar un trabajo y costear la mitad de los gastos de su educación.

Gráfico 2. Secuencia de eventos en la transición de Mariana

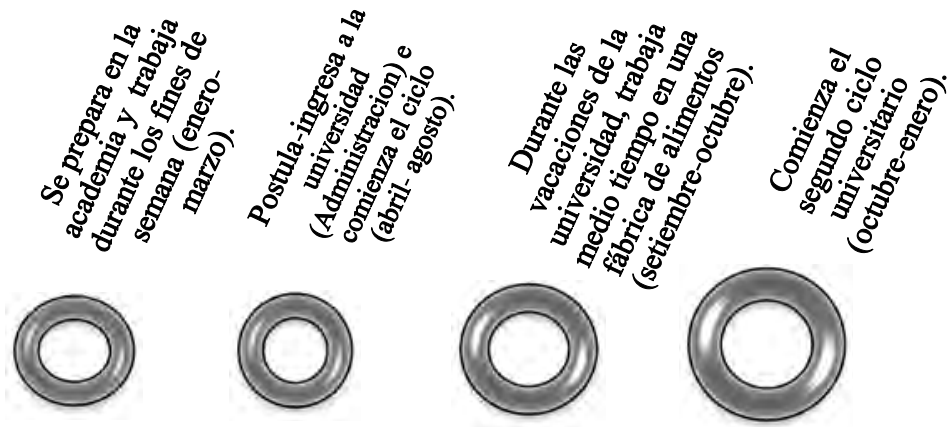

Durante la transición postsecundaria (año 2012)

Fuente: Elaboración propia

Como se observa, pese a la escasez de recursos de su familia, su madre costea la preparación de Mariana con la condición de que esta aproveche al máximo la oportunidad y ella consiga un trabajo los fines de semana que le sirva para cubrir sus gastos diarios. Ingresar a la universidad no le tomó mucho tiempo, debido a que, como señala, la academia le ayudó a sentir mayor presión y aprender rápidamente gracias a la competencia. Al ingresar, su vida diaria cambió, pues encontró mucha exigencia en los cursos y mayores posibilidades laborales en el futuro. Asimismo, una vez de vacaciones, consiguió un nuevo trabajo, a partir del cual asumió más responsabilidades vinculadas con las necesidades de su familia. Aunque reconoció que trabajaba porque lo necesitaba, también se dio cuenta de que lo hacía porque la ayudaba a sentirse más independiente dentro de los márgenes que su madre le permitía. Finalmente, Mariana se asumió como una persona activa y responsable en el proceso de continuar con sus estudios.

\subsection{Nadia: mayores seguridades cuando se goza del apoyo económico familiar}

Nadia es la cuarta de cinco hermanos. Todos viven con sus padres, quienes se dedican a la carpintería y al comercio. Sus padres llegaron a cursar la secundaria, pero solo su padre logró culminarla. Dos de sus hermanas mayores se encontraban estudiando una carrera profesional: una de ellas estudiaba en una universidad privada; y la otra, en el instituto pedagógico para docentes. Nadia 
comenta que su proyecto de vida se concreta al finalizar el colegio, después de evaluar distintas carreras. Un criterio importante para su elección profesional fue reconocer la rentabilidad económica. Siguiendo esta lógica, primero, quiso estudiar Ingeniería Ambiental y pensó que, si no ingresaba para esta carrera, postularía a Odontología «Ahora ya no se estudia porque me gusta, ahora se estudia porque tiene demanda [...]. Quise estudiar Ingeniería, porque ahora se necesita ingenieros y Odontología, porque tienes trabajo, ganas bien, puedes poner tu propio consultorio» (Nadia, 16).

Si bien la información recibida por parte de los profesores en el colegio fue bastante útil para decidirse por cuál carrera elegir, también le ayudaron los consejos de sus hermanas mayores (quienes se encontraban estudiando) y de sus tíos que son enfermeros, pero sobre todo de su padre y de su madre, pues ellos la apoyarían económicamente con los gastos de estudio. Como ya tenía los gastos educativos de sus otras hijas y no podía pagar una universidad privada, su padre le sugirió estudiar en una universidad pública. Sin embargo, en la universidad pública, Nadia no encontraba las carreras que, según ella, tienen mayor demanda. Por su lado, sus padres se sentían preocupados porque no quedaban muchas opciones profesionales para su hija. Para que Nadia "no pierda el tiempo" luego de terminar la secundaria, la convencieron de postular al instituto pedagógico y estudiar educación. Nadia confesó que hasta ese entonces no había pensado en la posibilidad de ser profesora, pero, viendo que por el momento no tenía otras opciones profesionales más convenientes, terminó el colegio y se preparó unos meses en la academia antes de postular al instituto pedagógico.

Gráfico 3. Secuencia de eventos en la transición de Nadia

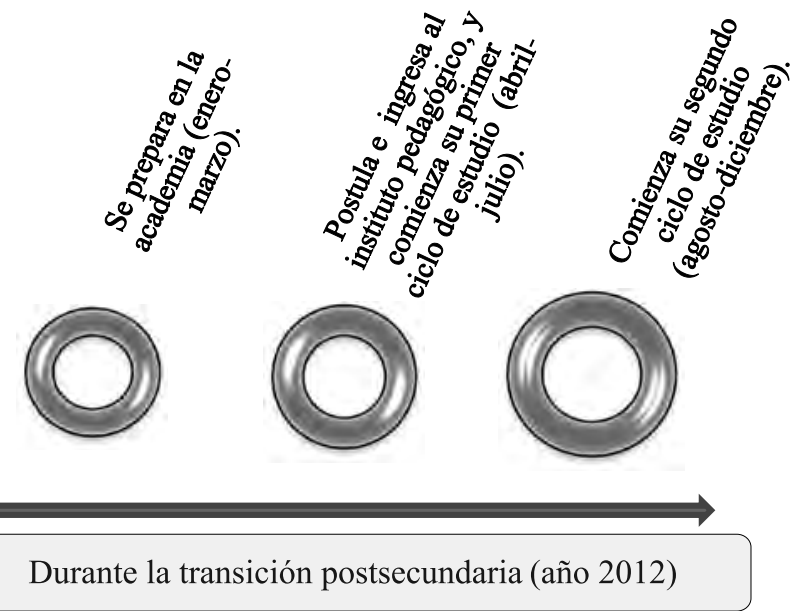

Fuente: Elaboración propia 
Como se observa, terminó el colegio, se preparó en la academia del instituto pedagógico, postuló e ingresó al poco tiempo. Nadia se considera una persona a la que le gusta estudiar, pero solo quiso seguir la carrera de Educación «mientras tanto» y hasta que pudiera conseguir la opción de Odontología. Reconoce que ser docente no es una profesión que tiene prestigio, pero que siempre habrá algún lugar donde trabajar. Sus padres quieren que solo estudie y que no se sienta obligada a trabajar para que pueda terminar su carrera en poco tiempo.

\subsection{Marisol: mayor incertidumbre por el futuro}

Marisol es la menor de cuatro hermanas. Vive con sus padres en una comunidad en Andahuaylas, donde se dedican a la agricultura y crianza de animales. Su padre tiene secundaria incompleta y su madre no culminó la primaria. De todas las hermanas, solo la mayor culminó la secundaria, mientras que las otras dos abandonaron el colegio tras quedar embarazadas.

Estudió la primaria y hasta el segundo de secundaria en el colegio de su comunidad. A partir de tercer año, se trasladó a un colegio en un ámbito más urbano, debido a que, según sus padres, en el colegio local, solo se hablaba en quechua y ellos querían que aprendiera el castellano. Aparte de estudiar, Marisol siempre participa ayudando en la chacra y en el cuidado de animales. Sin embargo, siente que sus padres le dan poca libertad y la controlan un poco. Lastimosamente, las experiencias de embarazo de sus hermanas mayores la condenan a vivir con la desconfianza de sus padres, motivo por el cual la controlan constantemente y le prohíben que salga a trabajar por temor a que se vincule con jóvenes de su edad y que cometa los mismos «errores» de sus hermanas: «yo quisiera apoyar a mis padres, quisiera trabajar como mis compañeras lo hacen, mi papá no me deja y yo quisiera ser profesional, pero no confían en mí» (Marisol, 17).

Frente a esta situación en su hogar, al llegar al quinto de secundaria, se sintió bastante insegura sobre sus posibilidades de estudiar una carrera profesional, principalmente, porque se sentía preocupada por la desconfianza de sus padres sobre su capacidad académica, más aún cuando le decían que no la apoyarían con sus estudios. Asimismo, menciona que sentía mucha preocupación, puesto que reconocía que tenía carencias económicas, pero, como había visto en otras jóvenes como ella, quería trabajar para ahorrar dinero y poder pagarse sus estudios. No pensaba estudiar en la universidad, debido a que sabía que le tomaría mucho tiempo desde la preparación hasta el ingreso. Por eso, apostó por estudiar en un instituto. 
Gráfico 4. Secuencia de eventos en la transición de Marisol

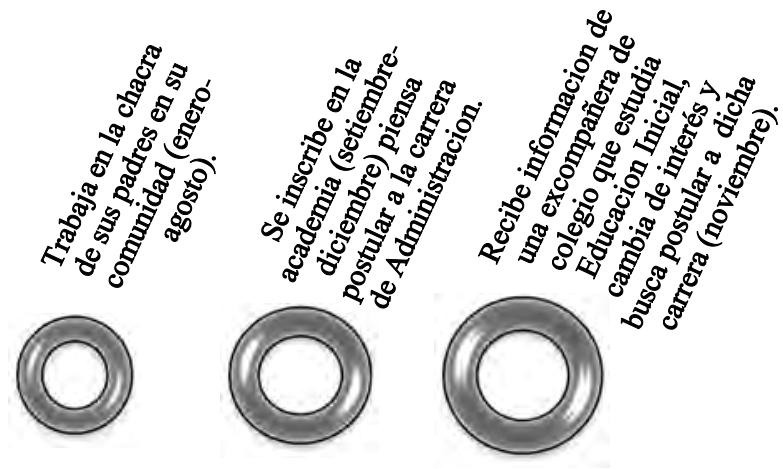

Durante la transición postsecundaria (año 2012)

Fuente: Elaboración propia

Tal cual se observa, muchas de las preocupaciones que Marisol tenía al finalizar el colegio se agudizaron con el paso de los meses. Al no lograr establecer un acuerdo con su padre y al no tener la libertad para conseguir un trabajo, no pudo estudiar durante la mitad del año posterior a la culminación de la secundaria. Solo después de varios meses, su padre le dio una oportunidad en los estudios. Se matriculó en la academia con la condición de estudiar lo que su padre había decidido por ella. Aunque Marisol aceptó las condiciones, en la academia, descubrió que no estaba preparada para ingresar a la universidad. En paralelo, una compañera de colegio le comentó sobre su experiencia estudiando Educación Inicial y a Marisol le comenzó a interesar esa opción. No obstante, continúa sintiéndose limitada e insegura para tomar sus decisiones, porque no cuenta con el respaldo de su familia.

\subsection{Fabián: inseguridades a pesar de contar con el apoyo económico necesario}

Fabián es el menor de siete hermanos. Vive con sus padres en su comunidad, quienes no pudieron culminar la secundaria. Sin embargo, los hermanos mayores de Fabián sí terminaron la secundaria, e, incluso, algunos son profesionales y viven entre Lima y Andahuaylas. Al ser el último de los hermanos, Fabián ha recibido el apoyo de sus padres y hermanos para culminar satisfactoriamente su escolaridad. Tener el respaldo económico para estudiar, sin embargo, ha significado recibir mayor presión para cumplir las expectativas de su familia. Durante el quinto año escolar, sus expectativas eran mudarse a vivir a Lima, trabajar y matricularse en una academia de fútbol. Luego de terminar el colegio, Fabián viajó a Lima y vivió en la casa de un hermano. Antes de salir de Andahuaylas, su madre y hermanos que estaban dispuestos a apoyarlo 
económicamente se negaron a darle permiso para viajar, aunque luego decidieron dejarlo ir, arguyendo que era importante «que experimente como es la vida allá». Fabián, quien antes había visitado Lima, planeó quedarse un tiempo para independizarse y lograr sus objetivos. Poco después de llegar a la capital, encontró un trabajo y ahorró para matricularse en la academia de futbol. Pese a que se matriculó en la academia, meses después decidió volver a Andahuaylas.

¿Qué le hizo cambiar de opinión para que retornara? Ya trabajando en Lima, Fabián se dio cuenta de que la vida es bastante dura y sacrificada. Pese a tener una casa donde llegar, sintió que mantenerse en la ciudad requería de bastante esfuerzo y que era poco probable alcanzar su proyecto inicial. Considerando que en Andahuaylas tenía el apoyo de su familia, decidió volver y prepararse en la academia para postular a la carrera de Administración en la universidad.

Gráfico 5. Secuencia de eventos en la transición de Fabián

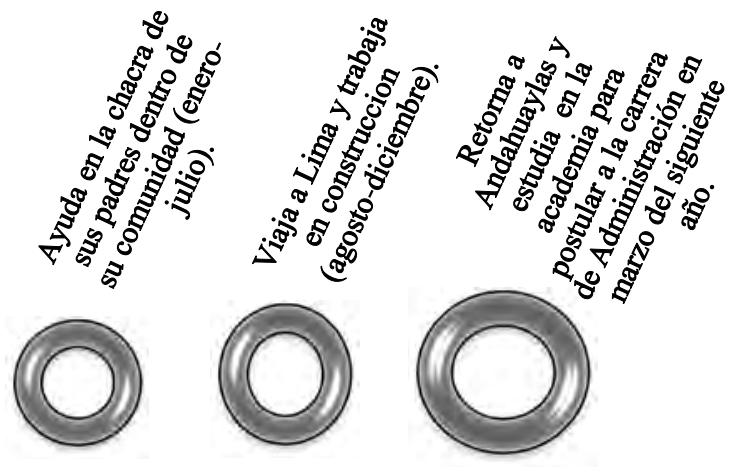

Durante la transición postsecundaria (año 2012)

Fuente: Elaboración propia

En el caso de Fabián, puede señalarse que sus inseguridades se debían al hecho de que las decisiones tomadas no eran propiamente académicas, como sí lo eran para sus demás compañeros. Por este motivo, no responder a las expectativas frecuentes de otros jóvenes y a lo esperado por la familia le generaban dudas. Ante esto, decidió quedarse en la chacra ayudando a la familia, mientras evaluaba qué estrategias emplear si es que ya no viviera en Andahuaylas.

\section{Cambios en las actitudes de los jóvenes y los proyectos familiares}

Para abordar los cambios que ocurren en las transiciones postsecundarias, se identificaron cuatro tipos de estrategias realizadas de manera diferenciada 
entre varones y mujeres. El análisis de las estrategias resulta crucial tomando en cuenta el papel de la agencia que proponen los estudios pioneros en el curso de vida (Elder, 1994; Heinz \& Krüger, 2001). Los sujetos toman decisiones dentro del marco de oportunidades y restricciones que les toca vivir (Sepúlveda, 2013).

Para este caso, las estrategias entran en diálogo con las expectativas y se interconectan con los factores educativos, económicos y culturales, previamente mencionados. Sobre esto, encontramos que, según el género, ocurren cambios significativos en los proyectos de vida de estos actores sociales. Dichos cambios evidencian tanto las posibilidades como las restricciones que viven los jóvenes, y se convierten en momentos críticos (Thomson et al., 2002) y en coyunturas vitales, especialmente, en el caso de las mujeres (Johnson-Hanks, 2002). De acuerdo con ello, encontramos las siguientes estrategias.

\subsection{Preparación en la academia}

La academia se convierte en el paso necesario para todos los postulantes a la educación superior, sobre todo, a las universidades públicas, pero incluso a algunos institutos que también requieren de una evaluación antes de ingresar. El ingreso a la academia no es una circunstancia, sino una decisión que se conecta con la evaluación de los factores económicos, culturales y educativos, previamente señalados. No obstante, siguiendo a Thomson et al. (2002), la experiencia en la academia se convierte en un momento crítico que transforma las expectativas de los jóvenes, ya sea para continuar o para dejar el camino de la universidad. Todos los jóvenes de los casos expuestos estuvieron en distintas academias con tiempo de preparación diferenciada; sin embargo, en el camino, algunos optaron por postular a un instituto pedagógico. Esta estrategia muestra que la transformación de las expectativas iniciales no agota las intenciones de continuar las trayectorias educativas (Pallas, 2003) y que, más bien, abre nuevas posibilidades que los jóvenes saben aprovechar teniendo el respaldo de sus familias (Punch, 2002).

\subsection{Estudiar «mientras tanto»}

«Mientras tanto» se entiende como aquello que uno en principio no decide, pero sucede y modifica las trayectorias educativas (Johnson-Hanks, 2002). Este hecho forma parte de las circunstancias que se originaron en el camino (Otero, 2010) y que es propio del margen de incertidumbre con el cual se lidia en el curso de vida (Elder, 1994). En este caso, el «mientras tanto» ocurre debido a factores como el tiempo y el respaldo económico familiar que debe aprovecharse tan pronto sea posible ${ }^{27}$. Sin embargo, más que evidenciar una situación

27. Varios de los jóvenes de la muestra tomaron una estrategia de este tipo, especialmente, aquellos que terminaron estudiando en el Instituto Pedagógico. Nadia, por 
de interdependencia de acuerdos entre padres e hijos (Punch, 2002), está sujeto a condiciones de apoyo diferenciados para los jóvenes varones y mujeres. Lo cierto es que, retomando el estudio de Olivera (2009), el «mientras tanto» también evidencia mecanismos de exclusión que limitan las posibilidades de decisión de las jóvenes mujeres. En contraste con los varones, para quienes ello implica mostrar mayor grado de independencia de los padres, para el caso de las mujeres, el control sobre la sexualidad genera mayores restricciones. Los padres que pueden invertir en la educación de sus hijas lo hacen para evitar que "pierdan el tiempo", se comprometan con la pareja y puedan salir embarazadas. Esta situación, en términos de Johnson-Hanks (2002), se vive como una coyuntura vital en la cual las mujeres afrontan mayores restricciones que afectan el desarrollo de su autonomía, a diferencia de las mayores oportunidades que encuentran los varones.

\subsection{Trabajar para estudiar}

Combinar trabajo con el estudio es una de las estrategias frecuentes (León \& Sugimaru, 2013) y que forma parte de las experiencias vitales de los jóvenes. Sin embargo, las secuencias de trabajo varían dependiendo del tiempo, las necesidades económicas y los permisos que no son los mismos para varones y mujeres (Otero, 2010). Dado que el trabajo se convierte en una estrategia que promueve un mayor nivel de autonomía, la experiencia laboral cobra un especial significado en esta etapa en la que es necesario tomar acciones sobre el proyecto de vida. Ahora bien, los hallazgos muestran que la capacidad de agencia que tienen los jóvenes se diferencia según sean varones o mujeres. Tal como Heinz y Krüger (2001) señalan, al interconectarse la agencia con las condiciones estructurales, los jóvenes varones desarrollan mayores grados de autonomía mediante el trabajo que las jóvenes mujeres. En este sentido, el control de la sexualidad de los padres sobre las hijas, al que alude Olivera (2009), permite que las jóvenes tengan mayores restricciones para salir a trabajar y, en caso de hacerlo, estén bajo supervisión de familiares cercanos.

\subsection{Permanecer en la provincia y aprovechar las oportunidades educativas locales}

La permanencia de los jóvenes en la provincia es una importante estrategia que corresponde a las altas valoraciones educativas de las familias (Benavides, Olivera \& Mena, 2006) frente a un contexto favorable para la educación superior y el creciente desarrollo comercial en la región. Ahora bien, el hecho de

ejemplo, reconoció que ser profesor otorga prestigio social y que siempre brinda trabajo. Por ello, su familia la apoyó para que estudiara en el pedagógico y para que, al terminar la carrera, buscara la manera de convalidar sus estudios en la universidad, que sí le garantizaría un mejor sueldo. 
que los jóvenes busquen ser profesionales en su localidad refleja directamente el aprovechamiento de las oportunidades estructurales del contexto en el que ven una posibilidad factible de lograrse, a diferencia de las posibilidades para acceder a la educación superior en otros lugares lejanos ${ }^{28}$. Más allá de ello, esta estrategia también refleja la interconexión de las decisiones sobre el marco de oportunidades que ofrece el contexto (Sepúlveda, 2013), y la interacción de los sujetos con la sociedad en niveles macro (de las oportunidades) y micro de las decisiones que toman (Ulrich, 2004), siempre sujetas a márgenes de posibilidad, como señala Thomson et al. (2002).

En síntesis, cada una de las cuatro estrategias ha sido producto de la interrelación de los factores económicos, educativos y culturales con los márgenes de incertidumbre asociados a los modos de apoyo diferenciados de los padres. En este sentido, recordando la propuesta de Elder (1994), y Heinz y Krüger (2001) sobre los sujetos que están inmersos entre estructuras y márgenes de posibilidad, se observa que, para el caso de las transiciones postsecundaria, las estrategias visibilizan una juventud activa que busca desarrollar mayores grados de autonomía. No obstante, las estructuras de oportunidad propias de la organización social del contexto pueden limitar a las mujeres a diferencia de los varones.

\section{Reflexiones finales}

En este artículo, se han expuesto las particularidades que ocurren en la transición postsecundaria a través de las expectativas y estrategias generadas, que -además- se transforman a lo largo del tiempo. De acuerdo con el curso de vida, vemos que la transición postsecundaria moldea y forma parte de una trayectoria educativa mayor en el sentido de la denominación de Pallas (2003) para referirse a los cambios actuales que afrontan las transiciones a la vida adulta como producto de la expansión educativa. Entre los hallazgos, encontramos que la búsqueda por acceder a los estudios superiores refleja una extensión de la trayectoria educativa y que, como toda trayectoria, involucra diversas transiciones.

Encontramos que la transición postsecundaria, pese a seguir una misma trayectoria, no es unívoca. A partir de los cincos casos de los jóvenes de la muestra, se evidencia que existen tiempos y modos de hacer ciertamente diferentes para los varones y mujeres. Estas particularidades conducen a identificar cambios en las transiciones, que también se visibilizan al momento de analizar las estrategias. Los cambios, a su vez, pueden constituirse como oportunida-

28 En el caso de Fabián, por ejemplo, se va a Lima, pero luego retorna a Andahuaylas después de unos cuantos meses, pues afirma que mantenerse en la capital requiere una mayor inversión entre gastos de alimentación, estudios, transporte, etc. Mantenerse en Lima supone un mayor costo de vida que puede terminar obstaculizando el proyecto profesional. 
des para desarrollar mayor autonomía para unos, pero, también, en momentos críticos, pueden traducirse en mayores restricciones y menor control sobre las situaciones para otros. Sin embargo, el peso de la organización social genera diferencia entre varones y mujeres: las mujeres tienen mayores restricciones para conducir sus transiciones, mientras que los varones gozan de mayor libertad para desarrollar su autonomía.

Finalmente, a pesar de que existan diferencias entre los jóvenes, los hallazgos de este artículo también permiten mostrar que, siendo diversa, la juventud constituye una etapa que se vive de manera activa y en la cual la participación dialoga con la estructura de oportunidad en el contexto. Por tanto, el ser joven deja de ser un período de espera del futuro. En este sentido, los proyectos de vida, que también son proyectos familiares, son los principales indicadores de las expectativas que tienen los jóvenes en la actualidad.

\section{Nota biográfica}

\section{Melissa Villegas Quispe}

Es licenciada en Antropología por la Pontificia Universidad Católica del Perú y estudiante de la maestría en Sociología por la misma universidad. Asimismo, es miembro del grupo de investigación Edades de la Vida y Educación del Vicerrectorado de Investigación de la PUCP con experiencia de investigación e interés en juventudes rurales, trayectorias educativas y laborales. 


\section{Referencias}

Abbott, A. (1997). On the concept of turning point. Comparative Social Research, 16.

Alemán, Alejandro (2015). La educación superior tecnológica superior en cifras. La República. Lima, 11 de agosto. Recuperado de http://larepublica. pe/sociedad/397670-la-educacion-superior-tecnologica-superior-encifras

Ames, P. (2013). Niños y niñas andinos en el Perú: Crecer en un mundo de relaciones y responsabilidades. Boletín del Instituto Francés de Estudios Andinos, 42(3).

Aramburú, C. E. (2014). Motivaciones de los postulantes seleccionados e ingresantes de Beca 18 que deciden no seguir la beca. Lima: Pronabec y Ministerio de Educación.

Benavides, M. (2006). Los desafíos de la escolaridad en el Perú: Estudios sobre los procesos pedagógicos, los saberes previos y el rol de las familias. Lima: Grade.

Benavides, M., Olivera, I. \& Mena, M. (2006). De papás y mamás a hijos e hijas: Las aspiraciones sobre el futuro y rol de las familias en las actividades escolares en el Perú rural. En M. Benavides (Ed.), Los desafíos de la escolaridad en el Perú: estudio sobre los procesos pedagógicos, los saberes previos y el rol de las familias (pp. 157-201). Lima: Grade.

Blanco, M. (2011). El enfoque de curso de vida. Orígenes y desarrollo. Revista Latinoamericana de Población, 5(8).

Cenaun-Inei (2010). II Censo Nacional Universitario. Lima.

Cotler, J. (2015). Educación superior e inclusión social: Un estudio cualitativo de los becarios del programa Beca 18. Lima: Ministerio de Educación, Pronabec. Serie estudios breves 7.

Dávila, O. (2005). Adolescencia y juventud: De las nociones a los abordajes. Última década (21), 83-104.

De la Cadena, M. (1988). Comuneros en Huancayo. Migración campesina a ciudades serranas. Lima: Instituto de Estudios Peruanos.

Díaz, J. (2008). Educación superior en el Perú: tendencias de la demanda y la oferta. En M. Benavides (Ed.), Análisis de programas, procesos y resultados educativos en el Perú. contribuciones empiricas para el debate (pp. 83-129). Lima: Grade.

Dubet, F. \& Martuccelli, D. (1997). En la escuela. sociología de la experiencia escolar. Buenos Aires: Losada.

Dubet, F. (2010). La sociología de la experiencia. Madrid: Editorial Complutense, Centro de Investigaciones Sociológicas. 
Elder, G. (1994). Time, Human Agency, and Social Change: Perspectives on the life course. Social Psychology Quarterly, 57(1).

Escale-Minedu (2015). Tendencias. Escale. Estadísticas de la Calidad Educativa. Recuperado de http://escale.minedu.gob.pe/tendencias

Guber, R. (2001). La etnografía: Método, campo y reflexibilidad. Bogotá: Norma.

Guerrero, G. (2013). ¿Cómo afectan los factores individuales y esolares la decisión de los jóvenes a postular a educación superior? Un estudio longitudinal en Lima, Perú. Lima: Grade.

Hernández, O. (2006). Proyecto de vida y desarrollo integral humano. Revista internacion Crecemos, 6(1-2). Recuperado de biblioteca. clacso.edu.ar/ar/libros/cuba/cips/caudales05/Caudales/ARTICULOS/ ArticulosPDF/07D050

Heinz, W. \& Krüger, G. (2001). Life course: Innovatios and challenges for social research. Current Sociology, 49(2).

Guber, R. (2001). La etnografía: Método, campo y reflexibilidad. Bogotá: Norma.

Guerrero, G. (2013). ¿Cómo afectan los factores individuales y esolares la decisión de los jóvenes a postular a educación superior? Un estudio longitudinal en Lima, Perú. Lima: Grade.

Hernández, O. (2006). Proyecto de vida y desarrollo integral humano. Revista internacion Crecemos, 6(1-2). Recuperado de biblioteca. clacso.edu.ar/ar/libros/cuba/cips/caudales05/Caudales/ARTICULOS/ ArticulosPDF/07D050

Johnson-Hanks, J. (2002). On the limits of life stages in ethnography: Toward a theory of vital conjunctures. American Anthropologist, New Series, 104(3).

Juárez, F. \& Gayet, C. (2014). Transiciones a la vida adulta en países en desarrollo. Anual Review Sociology, 40.

León, J. \& Sugimaru, C. (2013). Entre el estudio y el trabajo: las decisiones de los jóvenes peruanos después de concluir la educación básica regular. Avances de Investigación, 11. Lima: Grade.

Maxwell, J. (2005). Qualitative research design: An interactive approach. Thousand Oaks: Sage.

Olivera, I. (2010). Interrupción escolar y arreglos familiares: Aportes a los estudios de deserción escolar desde el análisis de la relacion familiaescuela. Revista Peruana de Investigacion Educativa, 1(2), 113-140.

Olivera, I. (2009). Juventud rural y lucha por la ciudadanía: Límites y posibilidades en los procesos de socialización. Anthropológica, 27(27), 7-24. 
Otero, A. (2010). Los avatares de la transición a la vida adulta: El papel de la educación y el trabajo en los recorridos juveniles. Revista de Trabajo Social y Ciencias Sociales, 59. Recuperado de https:/dialnet.unirioja.es/servlet/ articulo? codigo $=3341009$

Pallas, A. (2003). Educational transitions, trayectories and pathways. Handbook of the life course. New York: Springer Us.

Pérez, E. (2008). La nueva ruralidad en América Latina: avances teóricos y evidencias empíricas. Bogotá: Clacso.

Punch, S. (2002). Youth transitions and interdependent adult-child relations in rural Bolivia. Journal of Rural Studies, 18(2), 123-133.

Sepúlveda, L. (2013). Juventud como transición: Elementos conceptuales y perspectivas de investigación en el tiempo actual. Última Década, 21(39). Recuperado de http://www.scielo.cl/scielo.php?pid=S071822362013000200002\&script=sci_arttext

Thomson, R., Bell, R., Holland, J., Henderson, S., McGrellis, S. \& Sharpe, S. (2002). Critical moments: Choice, Chance and opportunity in young people's narratives of transition. Sociology 36(2), 335-354.

Ulrich, K. (2004). Whose Lives? How history,societies and institutions define and shape life courses. Research in Human Development, 1(3), 161-187. 